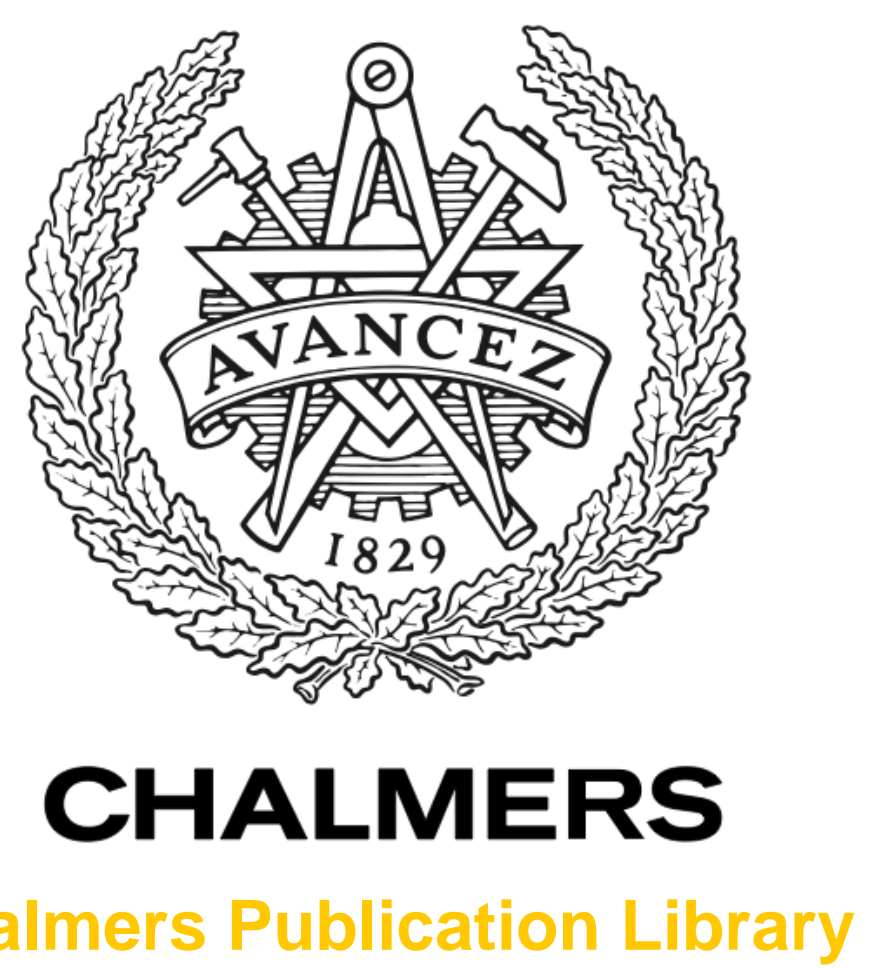

\title{
Terahertz transmission spectroscopy by Josephson oscillator and cold-electron bolometer
}

This document has been downloaded from Chalmers Publication Library (CPL). It is the author's version of a work that was accepted for publication in:

\section{SPIE USE}

Citation for the published paper:

Tarasov, M. ; Kuzmin, L. ; Stepantsov, E. (2004) "Terahertz transmission spectroscopy by Josephson oscillator and cold-electron bolometer". SPIE USE, vol. 4 pp. 5619.

Downloaded from: http://publications.lib.chalmers.se/publication/80932

Notice: Changes introduced as a result of publishing processes such as copy-editing and formatting may not be reflected in this document. For a definitive version of this work, please refer to the published source. Please note that access to the published version might require a subscription. 


\title{
Terahertz transmission spectroscopy by Josephson oscillator and cold-electron bolometer
}

\author{
M. Tarasov ${ }^{\text {a,b }}$, L. Kuzmin ${ }^{\text {b,d }}$, E.Stepantsov ${ }^{c, d}$, I.Agulo ${ }^{b}$, A.Kalabukhov ${ }^{\text {d,b }}$, A.Kidiyarova- \\ Shevchenko ${ }^{\mathrm{b}}$, T.Claeson ${ }^{\mathrm{b}}$ \\ ${ }^{a}$ Inst. of Radio Engineering and Electronics RAS, Mokhovaya 11, bld. 7, Moscow RU125009 \\ ${ }^{\mathrm{b}}$ Dept. of Microtechnology and Nanoscience, Chalmers Univ. of Technol., Göteborg, SE41296 \\ ${ }^{\mathrm{c}}$ Institute of Crystallography RAS, Moscow RU117333 \\ ${ }^{\mathrm{d}}$ Dep. of Physics, Moscow State University, Moscow RU119899
}

\begin{abstract}
For sensitive wideband spectroscopy at TeraHertz frequencies one needs a wide-range electrically tunable THz source and a sensitive detector. In this paper a superconducting normal metal cold electron bolometer (CEB) was used as a broadband sensor. Bolometers were integrated with broadband log-periodic antenna designed for $0.2-2 \mathrm{THz}$ frequency range and double-dipole antennas designed for 300 and $600 \mathrm{GHz}$ central frequency. A Josephson junction was used as a wide band electrically tuned terahertz cryogenic oscillator. Bicrystal $\mathrm{YBaCuO}$ Josephson junctions demonstrated a characteristic voltage $I_{c} R_{n}$ of over $4 \mathrm{mV}$ that corresponds to characteristic frequency about $2 \mathrm{THz}$. The bolometer chip is attached to a Si substrate lens at $260 \mathrm{mK}$ and the oscillator chip is attached to the sapphire substrate lens at $1.8 \mathrm{~K}$, with lenses facing each other at the distance of few centimeters. High signal was measured in the whole frequency range up to $1.7 \mathrm{THz}$ by simple changing the bias voltage of Josephson junction from zero to $3.5 \mathrm{mV}$. A voltage response of the bolometer up to $4 \cdot 10^{8} \mathrm{~V} / \mathrm{W}$ corresponds to an amplifier-limited technical noise equivalent power of the bolometer $\mathrm{NEP}=1.2510^{-17} \mathrm{~W} / \mathrm{Hz}^{1 / 2}$. Combining a Terahertz band Josephson junction, a high-sensitive hot electron bolometer, and a sample under test in between, makes it possible to develop a cryogenic compact Terahertz-band transmission spectrometer with a resolution below $1 \mathrm{GHz}$ corresponding to the linewidth of Josephson oscillations. For frequencies below $600 \mathrm{GHz}$ a conventional $\mathrm{Nb}$ shunted SIS junction can be used as Josephson oscillator.
\end{abstract}

Keywords: Terahertz spectrometers, Josephson junctions, cold electron bolometers, noise equivalent power

\section{CRYOGENIC DETECTORS}

It has become increasingly evident in the last few years that superconducting devices will play a major role in radiation detection and characterization of electromagnetic spectrum in terahertz frequency range. The critical difference between detection at terahertz frequencies and detection at shorter wavelengths lies in the low photon energies (1-10 meV). The ambient background thermal noise almost always dominates narrow-band signals requiring cryogenic cooling. The use of superconducting sensors has been further promoted through the recent development of ultra-low temperature coolers which no longer need liquid cryogens. Superconducting devices offer the prospect of fundamental thermodynamic or quantum limited sensitivity, spectral sensitive detection with ability to measure photons individually, ability to manufacture large arrays with modern thin-film technology. Ultimate NEP of a bolometer (see [1]) is determined by fundamental thermodynamics and is essentially the same for all types $N^{2} P^{2}=4 k_{b} T^{2} G$ in which $k_{b}$ is Boltsman constant, $T$ is electron temperature, $\mathrm{G}$ is thermal conductance. The main limitation for practical detector NEP is determined by a background power load $\mathrm{P}_{0}$ that gives $\mathrm{NEP}^{2}=2 \mathrm{P}_{0} \mathrm{k}_{\mathrm{b}} \mathrm{T}_{\mathrm{e}}$ [2]. At present the state-of-the-art practical bolometers demonstrate NEP below $10^{-17} \mathrm{~W} / \mathrm{Hz}^{1 / 2}$ at $0.1 \mathrm{~K}$. Main generic types of cold direct detectors are following:

Transition Edge Sensor (TES) is the most acknowledged type of superconducting bolometer. It consists of a thin superconducting film that can change its resistance under the incoming radiation power. Depending on the frequency range, such a bolometer can be integrated with $\mathrm{THz}$ band planar antenna, or attached to absorber film that interacts with the optical or X-ray radiation. For X-ray detectors at $100 \mathrm{mK}$ one of the best $\mathrm{NEP}=3 \cdot 10^{-18} \mathrm{~W} / \mathrm{Hz}^{1 / 2}$ was demonstrated in [3]. The dynamic range of TES detectors is strictly limited by dc heating power applied before real operation. Any

\footnotetext{
*Mikhail.Tarasov@mc2.chalmers.se; phone 46-31-7725173; fax 46-31-7723471
} 
attempt to increase the dynamic range lead to additional heating with unavoidable degradation of the sensitivity. Practical TES sensors sacrifice sensitivity to avoid saturation.

Superconducting Tunnel Junctions (STJ) of the Superconductor-Insulator-Superconductor (SIS) type, as well as Superconductor-Insulator-Normal metal (SIN) type can be used from microwave to X-ray wavebands. They are currently being developed as photon counting detectors. STJ converts the incident radiation energy into a population of excited charges whose number is proportional to the deposited energy and to the inverse of the superconducting gap. The superconducting electrode in which this conversion takes place serves as absorber. By measuring the tunnel current it is possible to estimate the incoming energy and frequency [4]. Due to the leakage current and quantum efficiency about unity, the best NEP is about $10^{-16} \mathrm{~W} / \mathrm{Hz}^{1 / 2}$.

Superconducting Hot Electron Bolometer (SHEB) is mainly used in HEB mixers as a relatively fast (up to $10 \mathrm{GHz}$ ) power meter of interfered signal and LO waves, and in general it is the same type of power detector, because at signal and LO frequencies it is too slow and can't multiply these components. It has a very low thermal capacitance and a large thermal conductance, and in this way it is optimized for speed, but not for sensitivity. This type of sensor can be optimized for direct detection, a so-called Hot Electron Direct Detector (HEDD). The theoretical estimations of NEP below $10^{-20} \mathrm{~W} / \mathrm{Hz}^{1 / 2}$ [5] seems to be very optimistic. Taking into account the background power load, HEDD NEP should be limited at the same thermodynamic level of above $10^{-18} \mathrm{~W} / \mathrm{Hz}^{1 / 2}$.

Normal metal Hot Electron Bolometer with Andreev mirrors (ANHEB) was proposed by M.Nahum and P.L.Richards [6] and consists of a thin normal-metal strip between superconducting electrodes. Low electron-phonon interaction at low temperatures together with Andreev reflection at the boundary of a normal metal and a superconductor prevent heat leakage form hot electrons to phonons and to the electrodes. A superconductor-insulator-normal metal (SIN) junction attached to the normal metal strip is used for temperature sensing. The best $\mathrm{NEP}=510^{-18} \mathrm{~W} / \mathrm{Hz}^{1 / 2}$ was achieved at 100 $\mathrm{mK}$ [7].

SINIS normal metal cold electron bolometer (CCNHEB or CEB) was proposed in [8] and experimentally demonstrated in [9]. As in all previous cases, the responsivity and noise equivalent power (NEP) of the bolometer are mainly determined by its electron temperature. To improve CCNHEB performance it was suggested using direct electron cooling of the absorber by a superconductor-insulator-normal metal (SIN) tunnel junction [10]. The effect of electron cooling was demonstrated in [11]. The CEB is essentially a nanorefrigerator that cools the electrons within a thin metal film by extracting the hottest electrons through SIN junctions. This effect is similar to that used in a thermoelectric cooler (Peltier effect). In contrast to TES, an unavoidable dc heating for electrothermal feedback is replaced by a deep electron cooling, removing all incoming power from the absorber to the next stage. Thereby the electron temperature is maintained at the minimum level below the phonon temperature independently on the relatively high power load. The refrigeration effect allows this detector to operate with high sensitivity under high power load. The response time is determined by the tunneling time of electrons which can be very fast ( 10ns).

NISIN hot electron bolometer [12] is completely complementary to SINIS bolometer and clarify difference between hot electron and cold electron bolometers. In NISIN case a photon assisted tunneling through the barrier heats the middle S electrode. Hot electrons are injected into a superconductor, they reduce the energy gap, which in turn increase the current through the junction.

Kinetic Inductance Bolometer (KID) is based on the fast change of the kinetic inductance in a superconducting strip when a pair breaking process reduces the superfluid density [13]. The basic principle of operation of KID is to measure the resonant frequency of a thin-film superconducting resonator, operating at about $5 \mathrm{GHz}$. Incoming photons break superconducting pairs in a nearby absorber, and create quasiparticles. When these quasiparticles diffuse into the resonator, its inductance changes and its resonant frequency shifts. The frequency shift is detected by monitoring the transmission or reflection phase, and this shift is proportional to the energy of the absorbed photon. According to [14] the NEP is determined by the quasiparticle generation-recombination noise and at $\mathrm{T} \sim 1 \mathrm{~K}$ it can be as small as $10^{-19}$ $\mathrm{W} / \mathrm{Hz}^{1 / 2}$, again without accounting for the background power load.

Among these six generic superconducting bolometers the most are operated at electron temperature that is equal or above the bath or phonon temperature, and only CEB works at reduced electron temperature. Moreover, the electron 
cooling allows extracting the heating power, which leads to increase of the saturation level. As a result the effect of the background power load is not so severe as for the rest of bolometer types.

\subsection{Noise Equivalent power for CCNHEB (CEB).}

A simple analytic analysis [15] gives a rough relation for the practically achievable voltage response to the incoming power $\mathrm{S}_{\mathrm{v}}=\mathrm{dV} / \mathrm{dP}$ in a current bias mode :

$S_{V}^{\max }=\frac{2 k_{b}}{e \sum v T_{e}^{4}}=100 \mathrm{~V} / \mu \mathrm{W}$

And more accurate numeric calculation brings even higher value $4 \cdot 10^{8} \mathrm{~V} / \mathrm{W}$. Taking the bolometer noise $\mathrm{V}_{\mathrm{n}}=6 \mathrm{nV} / \mathrm{Hz}^{1 / 2}$ one can obtain the NEP in a current bias mode

$\mathrm{NEP}_{\mathrm{V}}=0.75 \cdot 10^{-17} \mathrm{~W} / \mathrm{Hz}^{1 / 2}$

We can also roughly estimate the characteristics for voltage bias mode with electron cooling. The main power stream from phonons to electrons at $300 \mathrm{mK}$ is

$$
P_{P h-e}=\Sigma v T_{P h}^{5}=0.5 p W
$$

To remove such a power from the electron system it is necessary to apply a cooling current of the value at least

$$
I_{c}=\frac{e P_{P h-e}}{k_{b} T}=2.2 \cdot 10^{-8} \mathrm{~A}
$$

This cooling current is associated with a shot noise. If we take the theoretical value [15] for the current response $\mathrm{S}_{\mathrm{I}}=\mathrm{e} / 2 \mathrm{k}_{\mathrm{b}} \mathrm{T}=6 \cdot 10^{3} \mathrm{~A} / \mathrm{W}$ it brings the

$N E P_{I}=\sqrt{4 k_{b} T_{e} \sum v T_{P h}^{5}}=1.3 \cdot 10^{-17} \mathrm{~W} / \mathrm{Hz}^{1 / 2}$

From the two dependencies above one can also obtain the required noise equivalent power for a voltage bias mode of operation. It is determined by the thermal conductivity of the SIN junction.

\subsection{Readout electronics}

At present we use a room-temperature JFET operational amplifier electronics with the noise level over $3 \mathrm{nV} / \mathrm{Hz}^{1 / 2}$. This makes the main limitation to the NEP of receiver. The natural way of improvement is to use a SQUID as a current readout. Our recent result for SQUID with cryogenic bulk transformer is $35 \mathrm{fA} / \mathrm{Hz} 1 / 2$ [16]. Unfortunately the advantage of high sensitivity is combined with a complicated operation technique and a very high sensitivity to external interference.

Recent results in PHEMT cold semiconducting amplifiers [17] bring realistic alternative to SQUID. For commercial transistor ATF-35143 at T=380 mK it was obtained the noise temperature $100 \mathrm{mK}$, voltage noise $100 \mathrm{pV} / \mathrm{Hz}^{1 / 2}$, current noise $1 \mathrm{fA} / \mathrm{Hz}^{1 / 2}$. These characteristics are measured for source resistance $10 \mathrm{~kW}$ in the frequency range $1-4 \mathrm{MHz}$ which nicely fit to the requirements for frequency domain multiplexing readout for CEB bolometers. It should be noted that at very low frequencies DC-100 kHz the pHEMT even at cryogenic temperatures cannot compete with the noise temperature of Si JFET. Real advantages may be achieved at frequencies over $1 \mathrm{MHz}$.

\section{SAMPLES LAYOUT AND FABRICATION}

A general view on cold electron bolometer with capacitive coupling (CCNHEB) chip is presented in Fig. 1. One can see in the center a broadband log-periodic antenna for frequency range $0.1-2 \mathrm{THz}$, and double-dipole antennas for 300 and $600 \mathrm{GHz}$ to the left and to the right from the center. Besides above and below the central antenna there are two structures with additional SIN junctions for studies of electron cooling in SINIS structures. The first step of sample fabrication was thermal evaporation of $60 \mathrm{~nm}$ Au for fabrication of the normal metal traps and contact pads. The pattern for the traps and the pads were formed using photolithography. The next step was the fabrication of the tunnel junctions and the absorber. The structures were patterned by e-beam lithography and the metals were thermally evaporated using the shadow evaporation technique. The $\mathrm{Al}$ (superconductor) was evaporated at an angle of about $60^{\circ}$ up to a thickness of $65 \mathrm{~nm}$ and oxidized at a pressure of $10^{-1}$ mbar for 2 minutes. A $\mathrm{Cr} / \mathrm{Cu}(1: 1)$ absorber of a total thickness of $75 \mathrm{~nm}$ was 
then evaporated directly perpendicular to the substrate. The cooling junctions have a normal state resistance $\mathrm{R}_{\mathrm{N}}$ equal to $0.86 \mathrm{k} \Omega$, while the two inner junctions have $\mathrm{R}_{\mathrm{N}}$ equal to $5.3 \mathrm{k} \Omega$. The inner junctions have a simple cross geometry, where a section of the normal metal absorber overlaps the thin $\mathrm{Al}$ electrodes. The area of overlap, which makes to the area of each of the tunnel junction, is equal to $0.2 \times 0.3 \mu \mathrm{m}^{2}$. The structure of the outer junctions is such that the ends of the normal metal absorber overlap with a corner of each of the Al electrodes, which have a much larger area compared to the middle $\mathrm{Al}$ electrode. The area of each of these junctions is $0.55 \times 0.82 \mu \mathrm{m}^{2}$. The purpose of the larger area $\mathrm{Al}$ electrode is to give more space for quasiparticle diffusion compared to the middle Al electrode with simple cross geometry. In the described structure, the two outer and inner junctions have the $\mathrm{R}_{\mathrm{N}}$ equal to $0.85 \mathrm{k} \Omega$ and $5.4 \mathrm{k} \Omega$, respectively. The volume of the absorber was $0.18 \mu \mathrm{m}^{3}$.

A bias cooling current is applied through the outer junctions and the absorber. These tunnel junctions act as the cooling junctions, and therefore serve to decrease the electron temperature of the absorber. To determine the electron temperature, the voltage across the inner junctions is measured. A small current bias is applied to these junctions. The bias has to be optimal to obtain the maximum linear voltage response on temperature, and yet not too large so as to disturb the cooling process in the absorber.

High critical temperature Josephson junctions on tilted bicrystal sapphire substrates were fabricated in $\mathrm{YBaCuO}$ epitaxial films with c-axis inclined in $\langle 100\rangle$ direction by angle $14^{\circ}+14^{\circ}$. Films $250 \mathrm{~nm}$ thick were deposited by pulsed laser ablation on tilted sapphire bicrystal substrates covered by a $\mathrm{CeO}_{2}$ buffer layer. The critical temperature of the film was $\mathrm{T}_{\mathrm{c}}=89 \mathrm{~K}$ and $\Delta \mathrm{T}_{\mathrm{c}}=1.5 \mathrm{~K}$. Bicrystal Josephson junctions of width from 1.5 to $6 \mu \mathrm{m}$ demonstrated a characteristic voltage $I_{c} R_{n}$ of over $4 \mathrm{mV}$ at a temperature of $4.2 \mathrm{~K}$. This makes them promising candidates as oscillators for Terahertz frequency band applications.

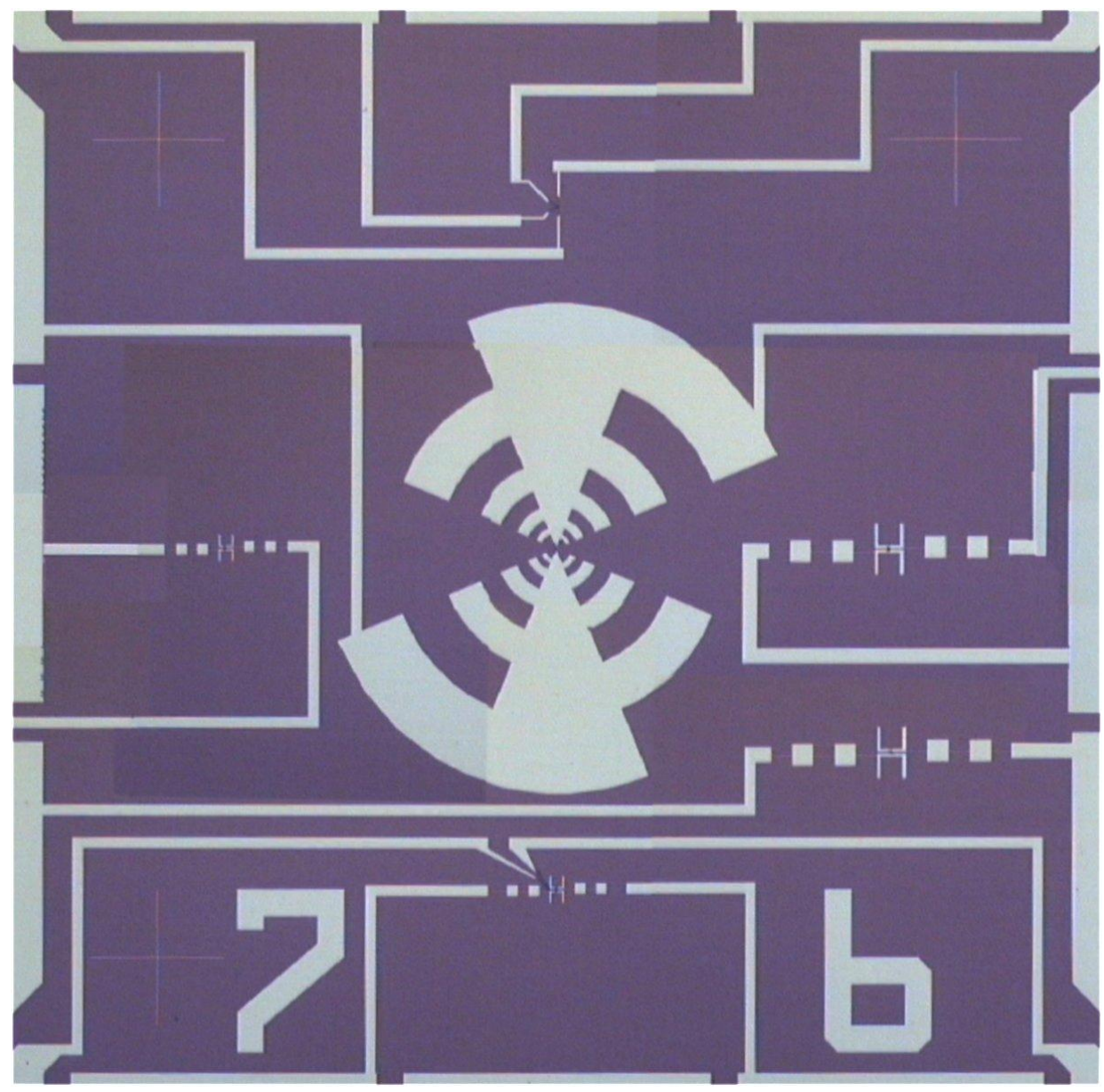

Figure 1. A bolometer chip layout. A wideband log-periodic antenna at the center, a $600 \mathrm{GHz}$ double-dipole antenna to the left and two $300 \mathrm{GHz}$ double-dipole antennas to the right. 


\section{POWER AND TEMPERATURE RESPONSES OF THE BOLOMETER.}

We measured the temperature response of the bolometers at the lowest temperature of about $260 \mathrm{mK}$ that is available in our He3 sorption cooler cryostat. The dc response was measured at upper and lower structures with four SIN junctions. Two external junctions were used as thermometers and two internal as heaters. The highest value of voltage response to temperature variations is over $1.6 \mathrm{mV} / \mathrm{K}$ and the largest current response about $37 \mathrm{nA} / \mathrm{K}$ for a $10 \mathrm{k} \Omega$ junction and $55 \mathrm{nA} / \mathrm{K}$ for a $6 \mathrm{k} \Omega$ junction.

It was possible to apply a dc power to the central pair of junctions and measure the response of the outer pair of SIN junctions for these samples with four SIN junctions. Results of current and voltage responses on dc power are presented in Fig. 2. We observed the largest voltage response of $400 \mathrm{~V} / \mu \mathrm{W}$ for a $70 \mathrm{k} \Omega$ junction and $550 \mathrm{~A} / \mathrm{W}$ for a $10 \mathrm{k} \Omega$ junction. The obtained values of current and voltage responses can be converted to the natural figure of merit for the sensitivity of the bolometer in terms of a Noise Equivalent Power (NEP).

$\mathrm{NEP}=\mathrm{I}_{\mathrm{n}} / \mathrm{S}_{\mathrm{i}}$ or $\mathrm{NEP}=\mathrm{V}_{\mathrm{n}} / \mathrm{S}_{\mathrm{v}}$

in which $I_{n}$ is the current noise, $V_{n}$ is the voltage noise, $S_{i}=d I / d P$ is the current response, $S_{v}=d V / d P$ is the voltage response of the bolometer. Taking the voltage noise of a room-temperature preamplifier about $3 \mathrm{nV} / \mathrm{Hz}^{1 / 2}$ one can obtain the technical TNEP value

$\mathrm{TNEP}=1.25 \cdot 10^{-17} \mathrm{~W} / \mathrm{Hz}^{1 / 2}$

Using measured values of the temperature response and the power response one can also obtain the thermal conductivity of the bolometer.

$G_{V}=\frac{\partial P}{\partial T}=\frac{\partial V / \partial T}{\partial V / \partial P}=0.8 \cdot 10^{-11} \mathrm{~W} / K$

Now we can calculate the thermodynamic NEP arising from the electron-phonon interaction $\mathrm{NEP}_{\mathrm{ep}}{ }^{2}=4 \mathrm{kT} \mathrm{T}^{2} \mathrm{G}$ in which thermal conductivity $\mathrm{G}=5 \Sigma \nu \mathrm{T}^{4}=10^{-11} \mathrm{~W} / \mathrm{K}, v$ is the absorber volume. This brings a thermodynamical noise equivalent power $\mathrm{NEP}_{\mathrm{TD}}=1.410^{-18} \mathrm{~W} / \mathrm{Hz}^{1 / 2}$, and if we compare with the thermal conductivity in the voltage bias mode it corresponds to a $\mathrm{NEP}_{\mathrm{V}}=1.3 \cdot 10^{-18} \mathrm{~W} / \mathrm{Hz}^{1 / 2}$.
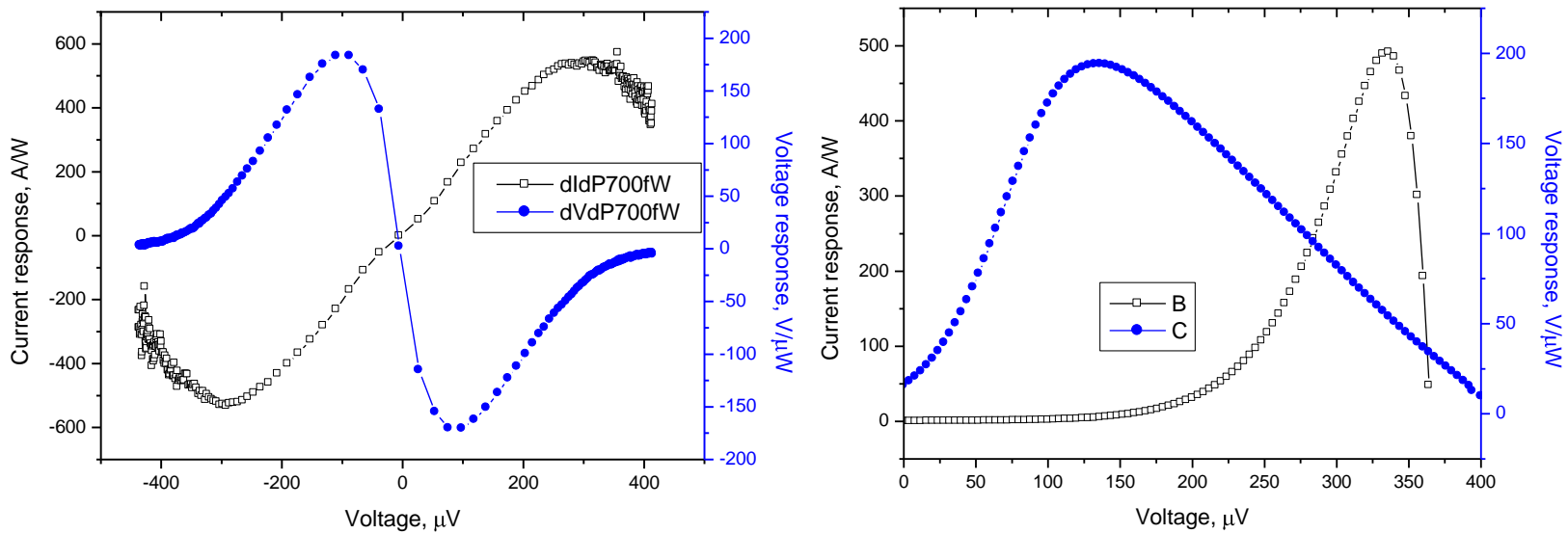

Fig. 2. Current and voltage responses for a $10 \mathrm{k} \Omega \mathrm{SIN}$ junctions to the applied dc power $700 \mathrm{fW}$ at $260 \mathrm{mK}$. Left panel experiment, right panel numerical modeling (sign for voltage response changed for easy comparison). 


\section{RESPONSE TO RADIATION FROM JOSEPHSON JUNCTION AT $260 \mathrm{mK}$}

In the first series of experiment we use a direct connection of the substrate with the Josephson oscillator to the substrate with the receiver. When a planar antenna is placed on a dielectric substrate with a high refraction index, the main lobe of the beam-pattern is directed into the substrate. In this case most of the radiation from the Josephson oscillator is directed to the antenna with the bolometer. The log-periodic antennas used in both oscillator and receiver chips are identical and designed for frequencies 100-2000 GHz.

The measured dependencies of the bolometer voltage response are presented in Fig. 3. Applying a magnetic field one can suppress the critical current of Josephson junction which leads to a decrease of the output power of Josephson oscillations and the frequency range according to the Josephson equations. When the critical current is suppressed below $2 \mu \mathrm{A}$ the response voltage is clear proportional to the square of the JJ bias current and is no more affected by magnetic field. It means that we completely suppressed Josephson radiation and the residual radiation is just a thermal radiation by overheated normal resistance of the Josephson junction matched to the broadband antenna. This brings clear evidence that we can separate the Josephson radiation at frequencies below $1 \mathrm{THz}$ and the thermal radiation of overheated matched load for bias voltages over $1 \mathrm{mV}$.

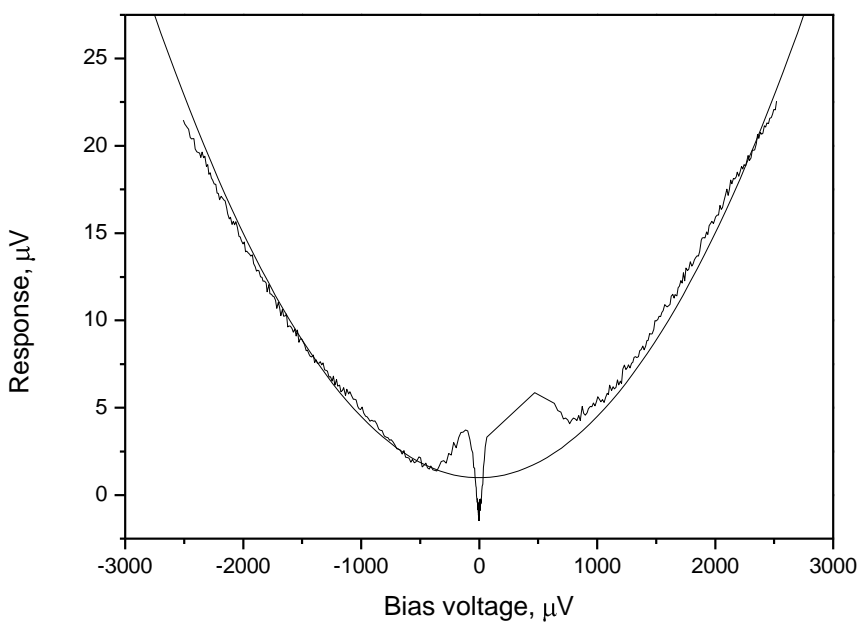

Figure 3. Bolometer response measured with a Josephson junction radiation source at $260 \mathrm{mK}$. The solid parabola is a fit for Joule heating.

For Josephson oscillators we can estimate the maximum available power as $\mathrm{P}_{\mathrm{osc}}=0.1 \cdot \mathrm{I}_{\mathrm{c}} \mathrm{V}_{\mathrm{c}}=2 \cdot 10^{-9} \mathrm{~W}$. Misalignment of antennas, mismatch of beam-patterns, material losses, mismatch of impedances can bring the total attenuation of the maximum power up to $30 \mathrm{~dB}$ that corresponds to an available power at the bolometer of about $10^{-12} \mathrm{~W}$. The estimated above bolometer responsivity is $\mathrm{S}=1.1 \cdot 10^{8} \mathrm{~V} / \mathrm{W}$ that brings the maximum voltage response to this power about $1.1 \cdot 10^{-4}$ $\mathrm{V}$. In our experiments we measured the voltage response up to $10 \mu \mathrm{V}$. The order of magnitude difference in response can be explained due to a non-ideal IV characteristic of the Josephson junction (excess current) and overheating that reduces the output power.

If we take as an approximation a model of overheating in a Josephson junction by [6] for a variable thickness microbridge

$T_{m}=\sqrt{T_{b}^{2}+3\left(\frac{e V}{2 \pi k}\right)^{2}}$

in which $\mathrm{T}_{\mathrm{b}}$ is bath temperature, $\mathrm{V}$ is a dc voltage bias, it brings the equivalent electron temperature at $1 \mathrm{mV}$ bias of about $3 \mathrm{~K}$. Taking into account that IR radiation is spread in a $4 \pi$ solid angle and the bolometer is at a distance of over 1 $\mathrm{mm}$, the dielectric can absorb a small part of this radiation, and the measured increase in temperature of $5 \mathrm{mK}$ looks reasonable. Now we should take into account that this power is radiated and then received. It means that Plank's radiation law should be applied 
$P_{r}=\frac{h f}{e^{\frac{h f}{k T}}-1} 0.3 f$

for which the maximum of radiation is obtained at $\mathrm{hf} \approx \mathrm{kT}$. If we apply the Plank's formula to equation (1) neglecting the phonon temperature, the approximation for moderate bias voltages is very simple:

$P_{\text {rad }}=\frac{0.6}{4 \pi^{2}} \cdot \frac{e^{2} V^{2}}{h}$

It brings the square-law voltage dependence, as observed in the experiment.

\section{IRRADIATION OF BOLOMETER BY A JOSEPHSON JUNCTION AT $1.8 \mathrm{~K}$}

To increase the output microwave power from the Josephson junction and increase the oscillation frequency it is necessary to increase the critical current of the Josephson junction. Placing the Josephson junction on the He4 stage prevents the sample from overheating by the relatively high power absorbed by the Josephson junction. Schematic view on experimental quasioptical setup is presented in Fig. 4. As the example if we take a junction with $10 \Omega$ normal resistance and oscillation frequency $300 \mathrm{GHz}$, it brings the absorbed power over $0.2 \mu \mathrm{W}$. At $1 \mathrm{THz}$ it is already $2.5 \mu \mathrm{W}$. The layout of the Josephson sample was the same as in the $260 \mathrm{mK}$ experiments with similar log-periodic antennas, but the critical current was over $500 \mu \mathrm{A}$ at $2 \mathrm{~K}$. As a result the $\mathrm{I}_{\mathrm{c}} \mathrm{R}_{\mathrm{n}}$ product exceeds $5 \mathrm{mV}$ for non-hysteretic junctions and such oscillators can in principle operate at frequencies over $2.5 \mathrm{THz}$. Experimental curves in Fig.5 measured by bolometers integrated with double-dipole and log-periodic antennas, and reveals that there is clear maximum at the design frequency $300 \mathrm{GHz}$ for DDA and smooth spectrum for LPA. The response at higher bias voltages for Josephson oscillator is presented in Fig. 6. The highest maximum corresponds to an oscillation frequency of $1.75 \mathrm{THz}$.

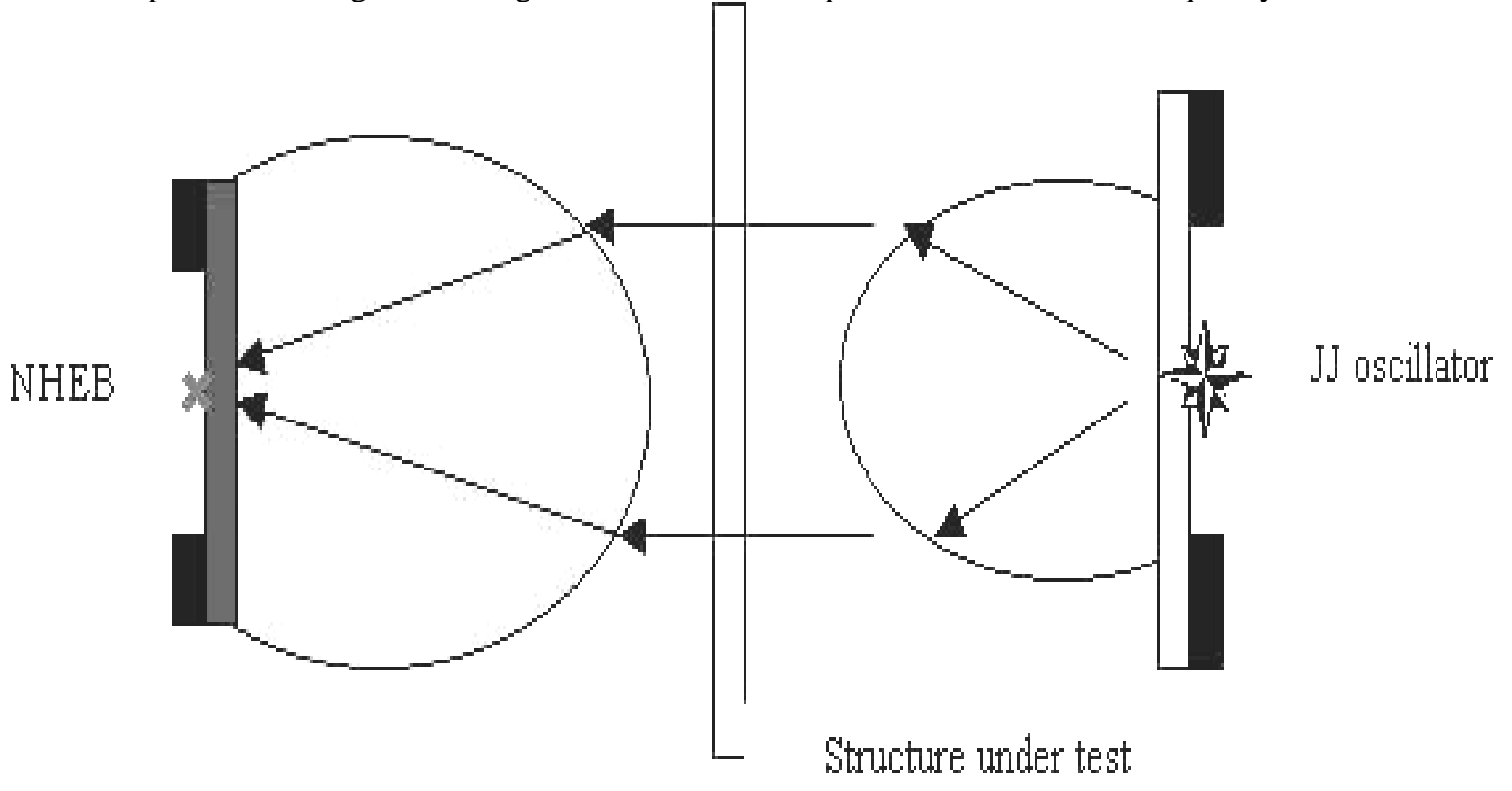

Fig. 4. Schematic view for quasioptical setup with NHEB lens-antenna unit at $260 \mathrm{mK}$ and Josephson junction oscillator lens-antenna unit at $1.8 \mathrm{~K}$. 


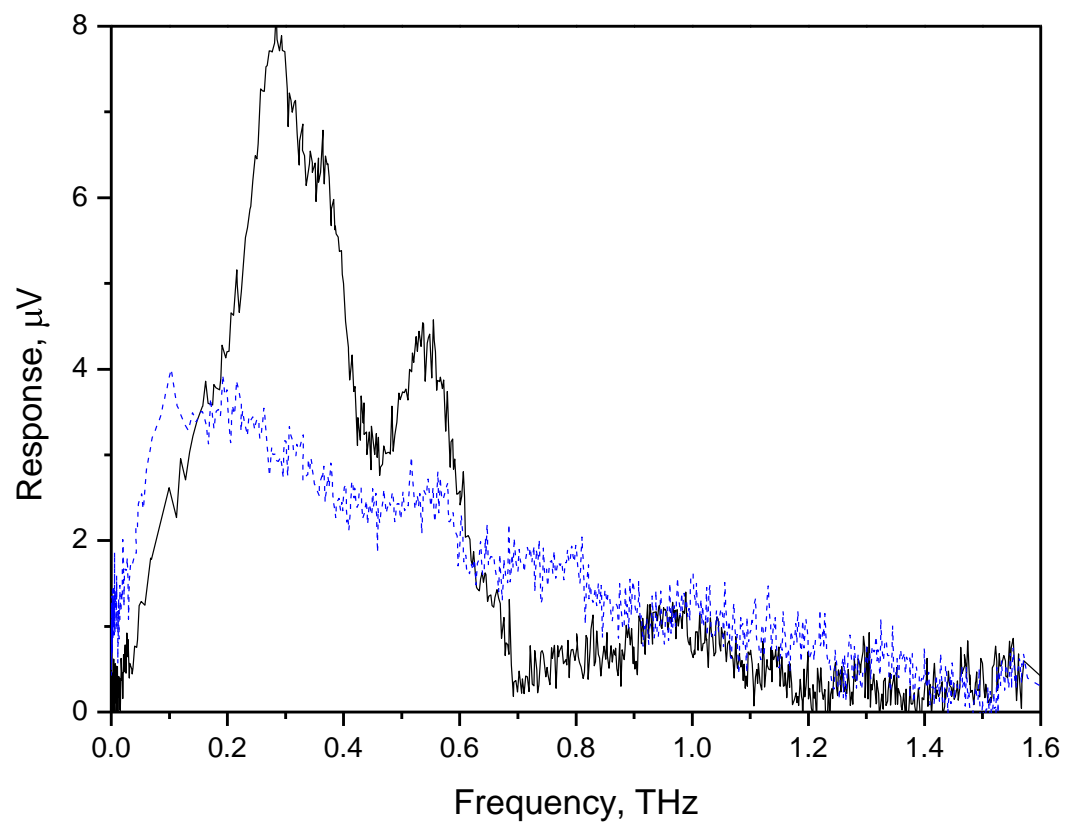

Figure 5. Response measured by double-dipole and log-periodic antennas for the same radiation source.

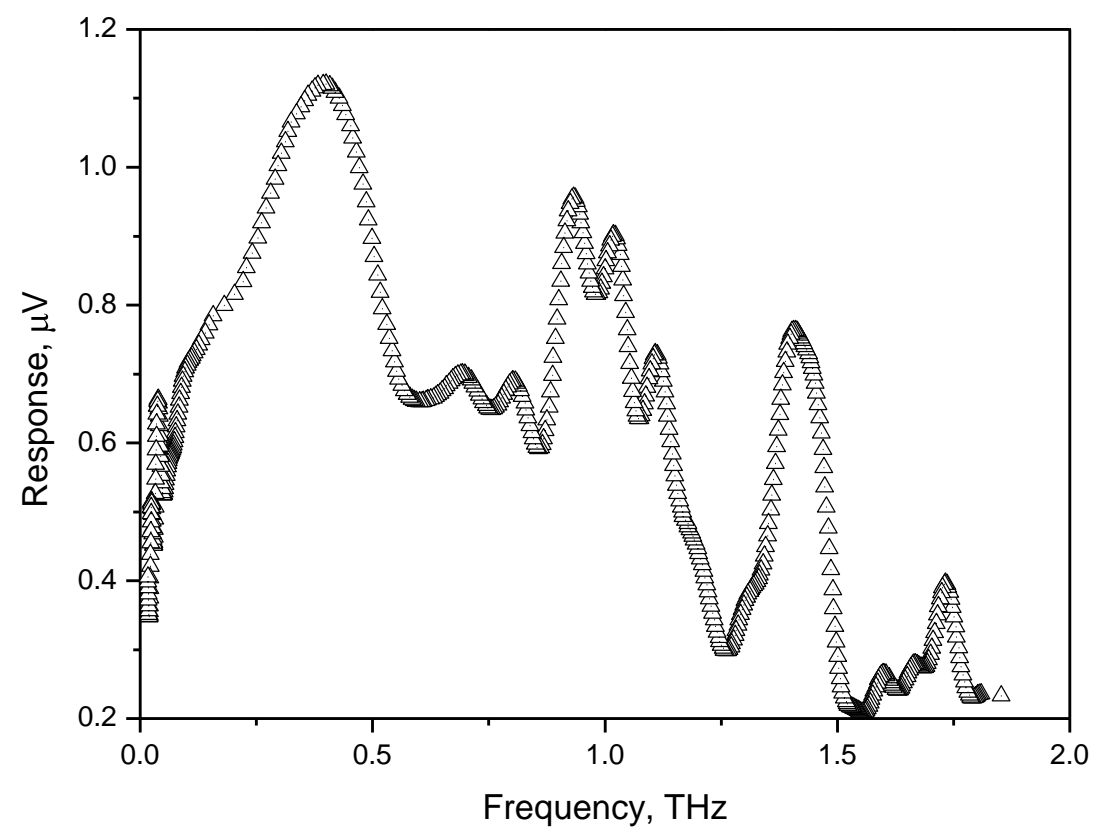

Figure 6. Response measured for high bias voltages of the Josephson junction. Last maximum corresponds to oscillation frequency $1.75 \mathrm{THz}$. 
For measurements at frequencies below $600 \mathrm{GHz}$ we use a low Tc Josephson oscillator. It is the same log-periodic antenna integrated with shunted Nb SIS tunnel junction. Samples were fabricated by HYPRES $3 \mu \mathrm{m}$ Nb process (for details see www.hypres.com). The linewidth of Josephson oscillations was measured irradiating such junction by a backward wave oscillator and monitoring the detector response. The selective detector response in Fig. 7 shows the voltage distance between bipolar maxima below $1 \mu \mathrm{V}$ that corresponds to the Josephson oscillations linewidth below 0.5 GHz.

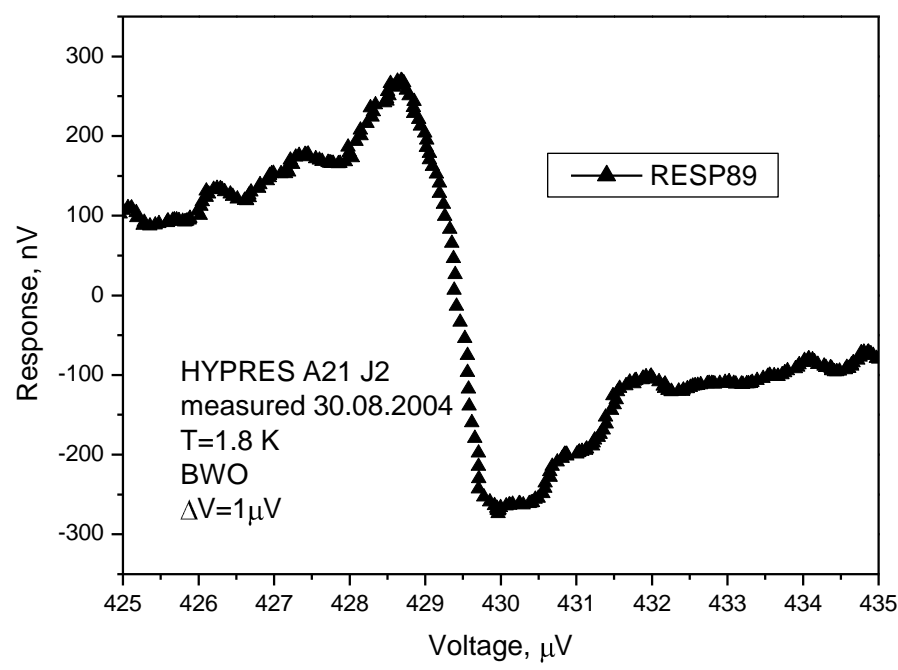

Figure 7. Selective detector response of $\mathrm{Nb}$ shunted tunnel junction at $215 \mathrm{GHz}$ with voltage distance between maxima about $1 \mu \mathrm{V}$ that corresponds to the linewidth $0.5 \mathrm{GHz}$.

\section{DISCUSSION}

Modern applications foreseen for terahertz spectroscopy are rapid scan and gas detection and identification systems (targeted molecule radars), terahertz detection of DNA signatures through dielectric resonances (phonon absorption). Direct competitors to superconducting spectrometer are huge and power consuming Fourier Transform Spectrometer (FTS) and Backward Wave Oscillator spectrometers. Tube sources require extremely high fields, both magnetic and electric. For comparison below is presented a table with main characteristics of two cryogenic spectrometers: with cyclotron emission from 2DEG composite bolometer (see [19]) and our Josephson-CEB spectrometer

\begin{tabular}{|c|c|c|}
\hline Main Characteristics & $\begin{array}{l}\text { Cyclotron emission from 2DEG - } \\
\text { composite bolometer detector }\end{array}$ & Josephson oscillator- CEB detector \\
\hline Spectral range & $0.1-2 \mathrm{THz}$ & $\begin{array}{r}0.1-1.7 \mathrm{THz}, \text { Josephson - up to } 4 \mathrm{THz} \\
\text { CEB - up to } 50 \mathrm{THz}\end{array}$ \\
\hline Spectral resolution & $200 \mathrm{GHz}$ & $1 \mathrm{GHz}$ \\
\hline $\begin{array}{l}\text { Emitted power } \\
\text { Received power } \\
\text { Bolometer sensitivity }\end{array}$ & $\begin{array}{l}1 \mathrm{~mW} \\
50 \mathrm{pW} \\
0.5 \mathrm{pW} / \mathrm{Hz}^{1 / 2}\end{array}$ & \begin{tabular}{|l}
$1 \mathrm{nW}$ \\
$1 \mathrm{pW}$ \\
$10^{-17} \mathrm{~W} / \mathrm{Hz}^{1 / 2}$
\end{tabular} \\
\hline Sweep & Magnetic feild & Voltage \\
\hline Modulation frequency & Few $\mathrm{Hz}$ & up to several $\mathrm{GHz}$ for Josephson oscillator \\
\hline
\end{tabular}


Development of cryogenic technique towards a "kitchen refrigerator" type that can be easy operated and does not require cryogen liquids and complicated process of operation opens the new possibilities for a wide application of cryogenic sensors. In Fig. 8 is presented a view of our experimental setup with a Oxford Instrument's cryogen-free He3 closed-cycle refrigerator HELIOX AC-V. The operation is simple: you press a button in the evening and have bolometer temperature about $280 \mathrm{mK}$ in the morning.

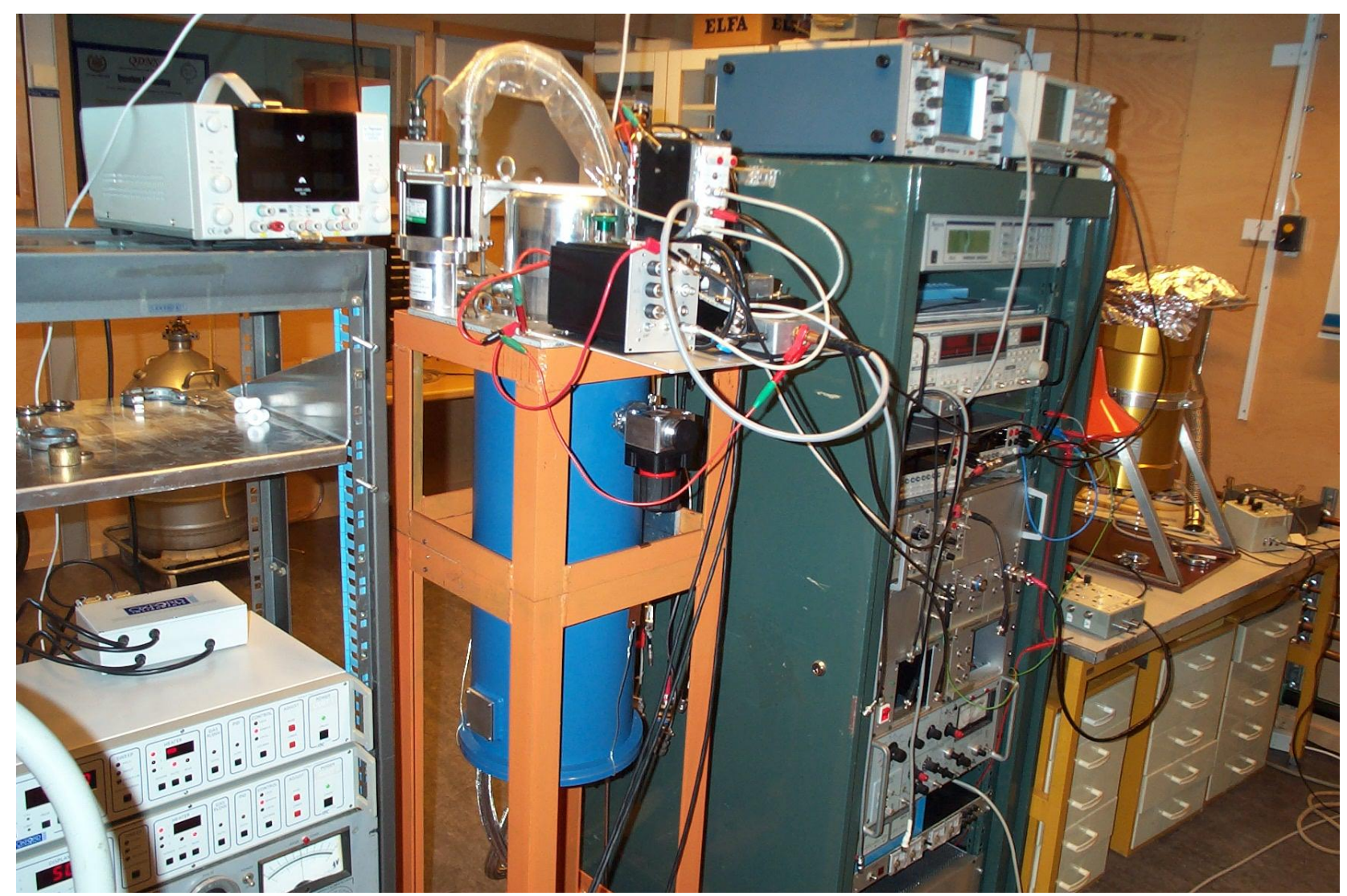

Figure 8. View of the experimental setup with HELIOX AC-V in the center and conventional INFRARAD Labs cryostat to the right.

\section{CONCLUSIONS}

We demonstrated the first experimental response of a normal metal cold electron bolometer at frequencies up to 1.7 THz. A voltage response of the bolometer is $410^{8} \mathrm{~V} / \mathrm{W}$ and an amplifier-limited technical noise equivalent power of the bolometer is $1.310^{-17} \mathrm{~W} / \mathrm{Hz}^{1 / 2}$. We use electrically tunable high critical temperature Josephson quasioptical oscillator as a source of radiation in the range $0.2-2 \mathrm{THz}$ and shunted $\mathrm{Nb}$ SIS Josephson junction for frequencies below $600 \mathrm{GHz}$. A high critical temperature Josephson junction operated at temperature about $2 \mathrm{~K}$ shows a $\mathrm{I}_{\mathrm{c}} \mathrm{R}_{\mathrm{n}}$ product over $4.5 \mathrm{mV}$ that enables an oscillation frequency over $2 \mathrm{THz}$. Combination of a Terahertz-band Josephson junction and a high-sensitive hot electron bolometer brings a possibility to develop a quasioptical cryogenic compact spectrometer with a resolution below $1 \mathrm{GHz}$. Such cryogenic spectrometer can be used for low-temperature spectral evaluation of biological and chemical samples in gas and solid states, cryogenic detectors, quasioptical submm wave grid filter, neutral density filter, absorber, etc. Cold electron bolometers can be used for remote atmosphere (gas) monitoring for pollution detection, etc.

\section{ACKNOWLEDGEMENTS}

Authors acknowledge support from VR, STINT, INTAS-01-686. 


\section{REFERENCES}

1. P.L.Richards, J. Appl. Phys., v. 76 (1), pp.1-24, 1994

2. L.Kuzmin, Proc. 15th Int. Symp. on Space Terahertz Technology, Northampton, April 27-29, 2004

3. K.D.Irwin, G.C.Hilton, D.A.Wollman, J.Martinis, Appl. Phys. Lett, 69 (13), pp. 1945-1947, 1996.

4. A.Peacock, P.Verhoeve, N.Rando, et al., J.Appl.Phys., 81 (11), 7641-7646, 1997.

5. M.E.Gershenson, D.Gong, T.Sato, B.S.Karasik, A.V.Sergeev, Appl. Phys. Lett., v. 79, N 13, pp. 2049-2051, 2001.

6. M.Nahum, P.L.Richards, C.A.Mears, IEEE Trans. Appl. Supercond., 3 (1), pp. 2124-2127, 1993.

7. D.Chouvaev, L.Kuzmin, Physica C, 352, pp. 128-130, 2001.

8. L.Kuzmin, Physica B 284-288, 2129, 2000.

9. M.Tarasov, M.Fominsky, A.Kalabukhov, L.Kuzmin, JETP Lett., 76, 507, 2002.

10. L.Kuzmin, I.Devyatov, D.Golubev, Proc. SPIE 3465, 193, 1998.

11. M.Nahum, T.M.Eiles, J.M.Martinis, Appl. Phys. Lett. 65, 3123, 1994.

12. R.Barends, J.R.Gao, T.M.Klapwijk, 6-th Eur. Workshop on Low Temp. Electronics (WOLTE-6), 23-25 June 2004, ESTEC, Noordwijk, The Netherlands, pp. 25-31.

13. E.Grossman, D.McDonald, J.Sauvageau, IEEE Trans Magn., V. 27, 2677-2680, 1991.

14. A.V.Sergeev, V.V.Mitin, B.S.Karasik, Appl. Phys. Lett., v. 80, N 5, pp. 817-819, 2002.

15. D.Golubev, L.Kuzmin, J. Appl. Phys., V. 89, N 11, P.1, pp.6464-6472, 2001.

16. M.Tarasov, A.Kalabukhov, S.Gudoshnikov, L.Kuzmin, O.Snigirev, S.Kovtonjuk, I.Lapitskaya, M.Kiviranta, H.Seppa, J. of Communications Technol. and Electronics, v. 48, N 12, 2003, pp. 1521-1526.

17. N.Oukhanski, H.-G.Meyer, Sixth European Workshop on Low Temp. Electronics (WOLTE-\&), 23-25 June 2004, ESTEC, Noordwijk, The Netherlands, pp. 163-168 (2004).

18. Tinkham M Octavio M Scocpol W J, JAP, v. 48, N 3, 1311-1320, 1977

19. Burke et al., Rev. Sci. Instr., 130-135, 2002. 